\title{
Truncated-distance codes and ternary compounds
}

\author{
I. Dejter • L. Fuentes · C. Araujo
}

Received: date / Accepted: date

\begin{abstract}
Here, perfect truncated-distance codes (PTDC's) in the $n$-dimensional grid $\Lambda_{n}$ of $\mathbb{Z}^{n}(0<n \in \mathbb{Z})$ and its quotient toroidal grids are obtained via the truncated distance $\rho(u, v)$ in $\mathbb{Z}^{n}$ given between vertices $u=\left(u_{1}, \cdots, u_{n}\right) \in$ $\mathbb{Z}^{n}$ and $v=\left(v_{1}, \ldots, v_{n}\right) \in \mathbb{Z}^{n}$ as the Hamming distance $d(u, v)$ in $\mathbb{Z}^{n}$ (or graph distance $d(u, v)$ in $\left.\Lambda_{n}\right)$ if $\left|u_{i}-v_{i}\right| \leq 1$, for all $i \in\{1, \ldots, n\}$, and as $n+1$, otherwise. While this $\rho$ is related to the $\ell_{p}$ metrics, the construction of PTDC's associated with lattice tilings of $\mathbb{Z}^{n}$ is obtained as that of rainbow perfect dominating sets in previous work. In this work, that construction is extended to that of ternary compounds $\Gamma_{n}$ obtained by glueing, or locking, ternary $n$-cubes along their codimension 1 ternary subcubes. We ascertain the existence of an isolated PTDC of radius 2 in $\Gamma_{n}$ for $n=2$ and conjecture that to hold for $n>2$ with radius $n$. Finally, we ask whether there exists a suitable notion replacing that of quotient toroidal grids of $\Lambda_{n}$ for the case of $\Gamma_{n}$.
\end{abstract}

Keywords truncated distance · truncated sphere · lattice tiling · binary cube $\cdot$ ternary cube

Mathematics Subject Classification (2010) 94B25 - 52C22 - 11H31 . 05C69

I. Dejter

University of Puerto Rico, Rio Piedras, PR

E-mail: italo.dejter@gmail.com

L. Fuentes

Universidad Nacional Abierta y a Distancia, Cartagena, Colombia

E-mail: luis.fuentes@unad.edu.co

C. Araujo

Universidad del Atlantico, Barranquilla, Colombia

E-mail: (carlosaraujo@mail.uniatlantico.edu.co 


\section{Introduction}

This work was motivated by computer architecture problems (cf 1]) associated to signal transmission in a finite field (cf. [10,11,14]). Related topics of coding theory and lattice domination are based on the Lee metric arising from the Minkowsky $\ell_{p}$ norm [4] with $p=1$. These themes, related to the still unsolved Golomb-Welch Conjecture [13,15, 16, were investigated via perfect Lee codes [10], diameter perfect Lee codes [14, tilings with generalized Lee spheres [2, 11], perfect dominating sets (PDS's) [20], perfect-distance dominating sets (PDDS's) [1] and efficient dominating sets [8].

Let $0<n \in \mathbb{Z}$. The aforementioned codes or dominating sets are generally realized in lattice tilings of $\mathbb{Z}^{n}$ whose tiles are translates of a common generalized Lee sphere. A natural follow-up here would be to consider lattice tilings of $\mathbb{Z}^{n}$ with two different generalized Lee spheres. Focus on such follow-up for $n>3$ is only possible by modifying the notion of the Lee distance $d$ to that of a truncated distance $\rho$, defined in the next paragraph (not a standard distance, but see Remark 5) and subsequently applied in Section 2 that restates results of [12]). Furthermore, new graphs $\Gamma_{n}$ built as compounds of ternary $n$-cubes, presented below in Remark 1 and Sections 3 that generalize the lattice graph of $\mathbb{Z}^{n}$, extend those applications.

Recall that the Hamming distance $h(u, v)$ between vectors $u=\left(u_{1}, \cdots, u_{n}\right)$ and $v=\left(v_{1}, \ldots, v_{n}\right)$ in $\mathbb{Z}^{n} \subset \mathbb{R}^{n}$ is the number of positions $i \in[n]=\{1, \ldots, n\}$ for which $u_{i} \neq v_{i}$. Now, let $\rho: \mathbb{Z}^{n} \times \mathbb{Z}^{n} \rightarrow \mathbb{Z}$ be given by:

$$
\rho(u, v)= \begin{cases}h(u, v), & \text { if }\left|u_{i}-v_{i}\right| \leq 1 \text { for all } i \in[n] ; \\ n+1, & \text { otherwise. }\end{cases}
$$

Given $S \subseteq \mathbb{Z}^{n}$, we denote by $[S]$ the induced subgraph of $S$ in the $n$ dimensional grid $\Lambda_{n}$, namely the graph whose vertex set is $\mathbb{Z}^{n}$ with exactly one edge between each two vertices at Euclidean distance 1 . In the above definition of $\rho$, the Hamming distance $h$ may be replaced alternatively by the graph distance in $\Lambda_{n}$ and is also our choice definition to be used on the graphs $\Gamma_{n}$ of Sections 3.4 .

To each component $H$ of $[S]$, we assign an integer $t_{H}>0$ to be understood in the context below as the radius of a truncated sphere with center $H$. For every component $H^{\prime}$ of $[S]$ in the translation class $\langle H\rangle$ of $H$ in $\mathbb{Z}^{n}$, we assume $t_{H^{\prime}}=t_{H}$. This yields a correspondence $\kappa$ from the set of translation classes $\langle H\rangle$ of components $H$ of $[S]$ into $\mathbb{Z}$ such that $\kappa(\langle H\rangle)=t_{H}$, for every $H \in\langle H\rangle$.

Let $\rho(u, S)=\min \{\rho(u, s) \mid s \in S\}$. Let $(H)^{\kappa(H)}=\left\{u \in \mathbb{Z}^{n} \mid \rho(u, H) \leq\right.$ $\kappa(H)\}$. A $\kappa$-perfect truncated-distance code, or $\kappa$-PTDC, is a set $S \subseteq \mathbb{Z}^{n}$ such that $\forall u \in \mathbb{Z}^{n}$ there exists a unique $s \in S$ with $\rho(u, s)=\rho(u, S)$ and such that the collection

$$
\left\{(H)^{\kappa(H)} \mid H \text { is a connected component of } S\right\}
$$

forms a partition of $\mathbb{Z}^{n}$. For each component $H$ of $S,(H)^{\kappa(H)}$ is referred to as the truncated sphere centered at $H$ (or around $H$ ) with radius $\kappa(\langle H\rangle)$. Here, 
$H$ is said to be the truncated center of $(H)^{\kappa(H)}$ and its vertices are said to be its central vertices. Given $0<t \in \mathbb{Z}$, a $\kappa$-PTDC is said to be a $t-P T D C$ if all $\kappa(\langle H\rangle)$ 's are equal to $t$. If $|H|=1$, then $\left|(H)^{t}\right|=\sum_{i=0}^{t} 2^{i}\left(\begin{array}{c}n \\ i\end{array}\right)$, for $0 \leq t \leq n$, (which is less than the cardinal of the corresponding $\ell_{1}$ sphere of radius $t$, if $t>1$ [4]).

Remark 1 A binary (resp. ternary) $n$-cube graph $Q_{n}^{i}$, succinctly called $n$-cube (resp. $n$-tercube), is a cartesian product $K_{i} \square \cdots \square K_{i}$ of $n$ complete graphs $K_{i}$ with $V\left(K_{i}\right)=\{0, \ldots, i-1\}=F_{i}$, the field of order $i=2$ (resp. 3). In Sections 3 . 4. we extend the study of PTDC's to edge-disjoint unions $\Gamma_{n} \supset$ $\Lambda_{n}$ of triangles; these are connected compounds of ternary $n$-cubes that we call ternary ( $n$-cube) compounds, (denomination that make us think of $\Gamma_{n}$ as binary (n-cube) compounds) and that cannot have non-isolated PDS's or non-isolated 1-PTDC's. (However, the ternary perfect single-error-correcting codes of length $\frac{3^{t}-1}{2}$ [18,2] are isolated $P D S$ 's, or efficient dominating sets [8] in the ternary $\frac{3^{t}-1}{2}$-cubes; these also are edge-disjoint unions of triangles, for $t>0)$. We will say for $G=\Gamma_{n}$ in Sections 34 that: an edge-disjoint union $G$ of triangles has a non-isolated $P D S S$ if every vertex of $G$ not in $S$ is adjacent to just either one vertex of $G$ (as in $P D S$ 's) or the two end-vertices of an edge of $G$. Section 4 shows that $\Gamma_{2}$ has an infinite number of isolated 2-PTDC and conjectures that $\Gamma_{n}$ has isolated $n$-PTDC's, $\forall n>2$.
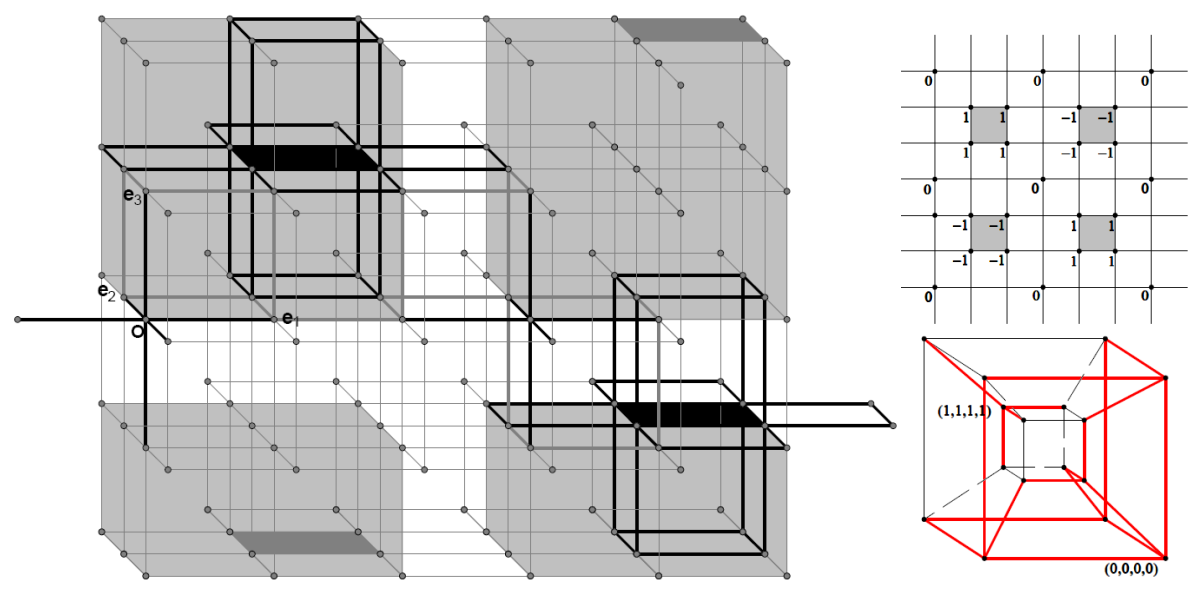

Fig. 1 Example accompanying Remark 2

Remark 2 We restate some results of [12] in Theorem 2, (resp. Theorems 34 4) below. They assert that PTDC's in $\Lambda_{n}$ that are lattice as in 14. (or latticelike [1]) exist having every induced component $H$ with vertex set (a) whose convex hull is an $n$-parallelotope (resp. either a 0 -cube or an $(n-1)$-cube) and (b) contained in a truncated sphere centered at $H$ with radius $n$ (resp. either 
$n-2$ or 1 ) forming part of the associated lattice tiling of $\mathbb{Z}^{n}$. In the center-left of Figure 1, such 0-cubes and $(n-1)$-cubes are suggested in black and darkgray colors for $n=3$ (Theorem 3), representing $\mathbb{Z}^{n}$ with dark-gray thin-edges and vertices, but dominating edges in thick black trace and other edges of a specific tile in thick dark-gray trace. This is schematized on the upper-right of the figure that represents the assignment of the last coordinate value mod 3 on the projection of this PTDC onto $\mathbb{Z}^{n-1}$. The case $n=4$ of Theorem 4 (the first PTDC which is neither PDS nor PDDS), can be visualized similarly. For example, the dominating edges, red colored in the copy of $Q_{4}^{2} \subset \mathbb{Z}^{4}$ in the lower-right of Figure 1 indicate a truncated sphere of radius 2 (resp. 1) at $(0,0,0,0)$ (resp. $(1,1,1,1))$. Also in the center-left of Figure 1 , light-gray 3 parallelotopes represent convex hull parts of truncated 3 -spheres whose central vertices form a lattice PTDC, the simplest case of Theorem 2. A coloring-graph viewpoint of these constructions is found in 12 .

Remark 3 (I) A t-PTDC (resp. a truncated $t$-sphere) of $\Lambda_{n}$ is defined like a PDDS [1] (resp. generalized Lee sphere [11]) with $\rho$ instead of the Lee distance d. (II) 1-PTDC's of $\Lambda_{n}$ (resp. truncated 1-spheres) coincide with PDS's [20] (resp. generalized Lee 1-spheres). (III) An example [1, also in Remark 7, illustrated in Figure 2 below, justifies that our definition of PTDC above employs translation classes instead of isomorphism classes, for in Figure 2 the truncated spheres have common radius (namely 1) for both translation classes, but that does not exclude the eventuality of an example with differing radii.

Remark 4 Motivation for Theorems 3,4 on building "lattice" $\kappa$-PTDC's whose induced components are $r$-cubes $(0 \leq r \leq n)$ of different dimensions $r$ comes from: (A) the perfect covering codes with spheres of two different radii in Chapter 19 [5] and (B) a negative answer [19] to a conjecture [20] claiming that the induced components of every 1-PTDC $S$ in an $n$-cube $Q_{n}^{2}$ are all $r$-cubes $Q_{r}^{2}$ (not necessarily in the same translation class [9]), where $r$ is fixed. In fact, it was found in 19] that a 1-PTDC in $Q_{13}^{2}$ whose induced components are $r$-cubes $Q_{r}^{2}$ of two different values $r=r_{1}$ and $r=r_{2}$ exists, specifically for $r_{1}=4$ and $r_{2}=0$. This seems to be the only known counterexample to the cited conjecture.

\subsection{Notation, $\ell_{p}$ metrics connections and periodicity}

If no confusion arises, every $\left(a_{1}, \ldots, a_{n}\right) \in \mathbb{Z}^{n}$ is expressed as $a_{1} \cdots a_{n}$. Let $O=$ $00 \cdots 0, e_{1}=10 \cdots 0, e_{2}=010 \cdots 0, \ldots, e_{n-1}=0 \cdots 010$ and $e_{n}=00 \cdots 01$.

Let $z \in \mathbb{Z}^{n}$, let $H=(V, E)$ be a subgraph of $\Lambda_{n}$ and let $H+z$ be the subgraph $H^{\prime}=\left(V^{\prime}, E^{\prime}\right)$ whose vertex set is $V^{\prime}=V+z=\left\{w \in \mathbb{Z}^{n} ; \exists v \in V\right.$ such that $w=v+z\}$ so that $u v \in E \Leftrightarrow(u+z)(v+z) \in E^{\prime}$. For example, let $H$ be induced in $\Lambda_{n}$ by the vertices with entries in $\{0,1\}$. Then, every translation $H+z$ of $H$ in $\Lambda_{n}$ (in particular $H$ itself) is isomorphic to $Q_{n}^{2}$.

Let $i \in[n]$. Each edge (resp. segment) $u v$ of $\Lambda_{n}$ (resp. $\mathbb{R}^{n} \supset \mathbb{Z}^{n}$ ) with $u \neq v$ is parallel to $O e_{i} \in E\left(\Lambda_{n}\right) \Leftrightarrow u-v \in\left\{ \pm e_{i}\right\}$ (resp. $\Leftrightarrow \exists a \in \mathbb{R} \backslash\{0\}$ 
with $\left.u-v=a e_{i}\right)$. A parallelotope $\mathcal{P}$ in $\mathbb{R}^{n}$ is a cartesian product of segments parallel to some of the $O e_{i}$ 's $(i \in[n])$. We restrict to $\mathcal{P}$ 's having their vertices in $\mathbb{Z}^{n}$. An edge of $\mathcal{P}$ is a segment of unit length parallel to some $O e_{i}(i \in[n])$ separating a pair of vertices of $\Lambda_{n}$ in $\mathcal{P}$. Recall that a trivial subgraph of $\Lambda_{n}$ is composed by just one vertex. The proof of the following is similar to that of Theorem 1 [1].

Theorem 1 Let $0<t \in \mathbb{Z}$. If $S$ is a $t$-PTDC in $\Lambda_{n}$, then the convex hull in $\mathbb{R}^{n}$ of the vertex set $V(H)$ of each nontrivial component $H$ of $[S]$ is a parallelotope in $\mathbb{R}^{n}$ whose edges are parallel to some or all of the segments $O e_{i}$ in $E\left(\Lambda_{n}\right)$, where $i \in[n]$.

Let $H$ be a nontrivial induced subgraph of $\Lambda_{n}$ with the convex hull of $V(H)$ in $\mathbb{R}^{n}$ as a parallelotope whose edges are parallel to some or all of the segments $O e_{i}$ in $E\left(\Lambda_{n}\right),(i \in[n])$. If exactly $r$ elements of $[n]$ are such values of $i$, then we say that $H$ is an $r$-box. Note that $H$ is a cartesian product $\prod_{i=1}^{n} P^{i}$, where $P^{i}$ is a finite path, $\forall i \in[n]$, with exactly $r$ paths $P^{i}$ having positive length. Clearly, the convex hull of an $r$-box is an $r$-parallelotope in $\mathbb{R}^{n}$. Also, every $r$-cube in $\Lambda_{n}$ is an $r$-box, for $r \in\{0\} \cup[n]$.

Let $W_{n, H, t}$ be the truncated sphere of radius $t$ around an $H$ as above, where $0 \leq t \in \mathbb{Z}$. Then, $H$ is the truncated center of $W_{n, H, t}$. A $t$-PTDC $S$ of $\Lambda_{n}$ determines a partition of $\mathbb{Z}^{n}$ into spheres $W_{n, H, t}$ with $H$ running over the components of $[S]$. Such an $S$ with the components of $[S]$ obtained by translations from a fixed finite graph $H$ is said to be a $t-P T D C[H]$. (This imitates the definition of a $t$-PDDS $[H]$ in [1], mentioned in Remark 3 (I) above). Let $S$ be a $t$-PTDC $[H]$ and let $H^{\prime}$ be a component of $[S]$ obtained from $H$ by means of a translation. Then $S$ is said to be a lattice $t-P T D C[H]$ if and only if there is a lattice $L \subseteq \mathbb{Z}^{n}$ such that: $H^{\prime \prime}$ is a component of $[S] \Leftrightarrow H^{\prime \prime}=H^{\prime}+z$, for some $z \in L$.

Remark 5 It is relevant to note the connections of $\rho$ and the $\ell_{p}$ metrics [4]: A truncated sphere of truncated radius 1 centered at some vertex $v$ is a Lee $(p=1)$ sphere of radius 1 , whereas a truncated sphere of truncated radius $n$ centered at $v$ is a sphere of radius 1 in the maximum $(p=\infty)$ distance, namely $W_{n,\{v\}, 1}$ (which has an $n$-dimensional cube for convex hull). Thus, all truncated spheres in this work are spheres in some $\ell_{p}$ metric, and the truncated distance is a convenient way to consider different $\ell_{p}$ metrics for the graph components. (When just one truncated radius $t$ is considered, the truncated distance is properly a distance). This observation allows connections with other previous works such as (for just one $t$ ) perfect codes in the maximum distance and in the $\ell_{p}$ metric [4].

Remark 6 To finish this subsection, we rephrase graph-coloring results of [12].

Announced as Theorem 2 below, there is a construction of lattice $n$-PTDC's $S$ whose induced subgraphs $[S]$ in $\Lambda_{n}$ have their components $H$ with:

(i) vertex sets $V(H)$ whose convex hulls are $n$-boxes in $\mathbb{R}^{n}$ with distance 3 to $[S] \backslash H$, and representatives (one per component $H$ ) forming a lattice with generators along the coordinate directions; 
(ii) each $V(H)$ contained in a truncated sphere $(H)^{\kappa(H)}$ whose radius is $n$.

(We do not know whether similar lattice $t$-PTDC's $S$ exist with $[S]$ having $r$-parallelotopes as their components, for $r$ fixed such that $0<r<n$.)

Extending the meaning of the adjective lattice in Subsection 1.2 below, as in 12 we have in Theorems 34 that a lattice $\kappa$-PTDC $S$ exists in $\Lambda_{n}$ whose induced subgraph $[S]$ has its components $H$ with vertex sets $V(H)$ :

(i') having both $(n-1)$-cubes and 0 -cubes as their convex hulls in $\mathbb{R}^{n}$;

$\left(\right.$ ii $\left.^{\prime}\right)$ contained in truncated spheres $(H)^{\kappa(H)}$ whose radii, namely 1 and $(n-2)$, correspond respectively to the $(n-1)$-cubes and 0 -cubes in item $\left(\mathrm{i}^{\prime}\right)$.

A set $S \subset \mathbb{Z}^{n}=E\left(\Lambda_{n}\right)$ is periodic if and only if there exist $p_{1}, \ldots, p_{n}$ in $\mathbb{Z}$ such that $v \in S$ implies $v \pm p_{i} e_{i} \in S, \forall i \in[n]$. Since each lattice $t$-PTDC $[H]$ $S$ is periodic [1, then every canonical projection from $\Lambda_{n}$ onto a toroidal grid $\mathcal{T}$, i.e. a cartesian product $\mathcal{T}=C_{k_{1} p_{1}} \square C_{k_{2} p_{2}} \square \cdots \square C_{k_{n} p_{n}}$ of $n$ cycles $C_{k_{i} p_{i}}$ $\left(0<k_{i} \in \mathbb{Z}, \forall i \in[n]\right)$, takes $S$ onto a $t$-PTDC $[H]$ in $\mathcal{T}$. This observation adapts to respective situations that complement the statements of Theorems 2 , 4 via canonical projections from $\mathbb{Z}^{n}$ onto adequate toroidal grids $\mathcal{T}$.

Question 1 Do lattice $t$-PTDC's $S$ exist with $[S]$ having $r$-parallelotopes as their components, for $r$ fixed such that $0<r<n$ ?

\subsection{Further specifications}

Given a lattice $L$ in $\mathbb{Z}^{n}$, a subset $T \subseteq \mathbb{Z}^{n}$ that contains exactly one vertex in each class mod $L$ (so that $T$ is a complete system of coset representatives of $L$ in $\Lambda_{n}$ ) is said to be an FR (acronym suggesting "fundamental region") of $L$. A partition of $\mathbb{Z}^{n}$ into FR's of $L$ is said to be a tiling of $\mathbb{Z}^{n}$. Those FR's are said to be its tiles.

We extend the notion of lattice $\kappa$-PTDC so that the associated fundamental region (see 3, pg 26), or FR (see above) of every new lattice contains a finite number of (in our applications, just two) members $H$ of each $\langle H\rangle$.

In the literature, existing constructions of lattice $t$-PTDC's in $\Lambda_{n}(t<n)$ concern just $t=1$ (see [1,2,11,14]) but there are not many known lattice 1-PTDC's. For example, [7] shows that there is only one lattice 1-PTDC $\left[Q_{2}^{2}\right]$ and no non-lattice 1-PTDC $\left[Q_{2}^{2}\right]$. In addition, there is a lattice $2-\operatorname{PDDS}\left[Q_{1}^{2}\right]$ in $\Lambda_{3}$ arising from a tiling of Minkowsky cited in [1].

Conjecture 1 There is no $t$-PTDC lattice in $\Lambda_{n}$, for $1<t<n$.

This conjecture has an analogous form for perfect codes in the $\ell_{p}$ metrics in [4], and together with the conjecture mentioned in Remark 4(B), produces a contrast with the constructions in Theorems 3, 4, below.

If $S$ is a periodic non-lattice $t$-PTDC $[H]$ in $\Lambda_{n}$, then there exists $0<m \in \mathbb{Z}$ and a tiling of $\Lambda_{n}$ with tiles that are disjoint copies of the vertex set $V\left(H^{*}\right)$ of a connected subgraph $H^{*}$ induced in $\Lambda_{n}$ by the union of: 
(a) the vertex sets of $m$ disjoint copies $H^{1}, \ldots, H^{m}$ of $H$ (components of $[S]$ );

(b) the sets $\left(H^{j}\right)^{\kappa(H)}$ of vertices $v \in \mathbb{Z}^{n}$ with $\rho\left(v, H^{j}\right) \leq t$, for $j \in[m]$, where $\left(H^{1}\right)^{\kappa(H)}, \ldots,\left(H^{m}\right)^{\kappa(H)}$ are pairwise disjoint copies of $(H)^{\kappa(H)}$ in $\mathbb{Z}^{n}$.

By taking such an $m$ as small as possible, we say that $S$ is a $t-P T D C[H ; m]$.

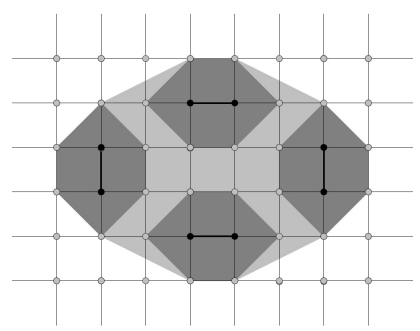

Fig. 2 FR of a lattice $L_{S}$ for a $1-\mathrm{PTDC}\left[Q_{1}^{2} ; 4\right] S$ in $\Lambda_{3}$.

Remark 7 In Section 5 1, a non-lattice 1-PTDC $\left[Q_{1}^{2} ; 4\right] S$ is shown to exist with a lattice $L_{S}$ based on it, each of the FR's of $L_{S}$ containing two copies of $Q_{2}^{2}$ parallel to $O e_{1}$ and two more copies of $Q_{2}^{2}$ parallel to $O e_{2}$, these four copies being components of $[S]$. This is represented in Figure 2, where the convex hulls of the truncated 1-spheres of such four components (in thick black trace) are shaded in dark-gray color and the remaining area completing their convex hull is shaded in light-gray color. A fixed vertex $v_{T}$ can be taken in each resulting tile $T$ so that all the resulting vertices $v_{T}$ constitute $L_{S}$.

Thus, even for a non-lattice $t$-PTDC $S$ in a $\Lambda_{n}$, a lattice can be recovered and formed by selected vertices $v_{T}$ in the corresponding tiles $T$ associated with $S$. We say that such set $S$ is a lattice $t-P T D C[H ; m]$, indicating the number $m$ of isomorphic components of $[S]$ in a typical tile $T$ in which to fix a distinguished vertex $v_{T}$.

We further specify the developments above as follows. A $t$-PTDC $S$ in $\Lambda_{n}$ with the components of $[S]$ obtained by translations from two non-parallel subgraphs $H_{0}, H_{1}$ of $\Lambda_{n}$ is said to be a $t$-PTDC $\left[H_{0}, H_{1}\right]$. The case of this in Figure 2 clearly is a $\kappa$-PTDC, with two translation classes of components of $[S]$ formed by the copies of $Q_{2}^{2}$ parallel to each of the directions coordinates. Here, $\kappa$ sends those copies onto $t=1$. Each tile of this $\kappa$-PTDC contains two copies of $Q_{2}^{2}$ parallel to $O e_{1}$ and two copies of $Q_{2}^{2}$ parallel to $O e_{2}$, accounting for $m=4$.

More generally, let $t_{i} \in[n]$, for $i=0,1$. We say that a set $S \subset V$ is a $\left(t_{0}, t_{1}\right)-P T D C\left[H_{0}, H_{1}\right]$ in $\Lambda_{n}$ if for each $v \in V$ there is:

$\left(\mathbf{i}^{\prime \prime}\right)$ a unique index $i \in\{0,1\}$ and a unique component $H_{v}^{i}$ of $[S]$ obtained by means of a translation from $H_{i}$ such that the truncated distance $\rho\left(v, H_{v}^{i}\right)$ from $v$ to $H_{v}^{i}$ satisfies $\rho\left(v, H_{v}^{i}\right) \leq t_{i}$ and

(ii' $\left.{ }^{\prime \prime}\right)$ a unique vertex $w$ in $H_{v}^{i}$ such that $\rho(v, w)=\rho\left(v, H_{v}^{i}\right)$. 
Even though such a set $S$ is not lattice as in [14] (or lattice-like [1]), it may happen that there exists a lattice $L_{S} \subset \mathbb{Z}^{n}$ such that for $0<m_{0}, m_{1} \in \mathbb{Z}$ there exists an FR of $L_{S}$ in $\Lambda_{n}$ given by the union of two disjoint subgraphs $H_{0}^{*}, H_{1}^{*}$, where $H_{i}^{*}(i=0,1)$ is induced in $\Lambda_{n}$ by the disjoint union of:

$\left(\mathbf{a}^{\prime}\right)$ the vertex sets of $m_{i}$ disjoint copies $H_{i}^{1}, \ldots, H_{i}^{m_{i}}$ of $H_{i}$, (components of $[S])$

$\left(\mathbf{b}^{\prime}\right)$ the sets $\left(H_{i}^{j}\right)^{\kappa(H)}$ of vertices $v \in \mathbb{Z}^{n}$ for which $0<\rho\left(v, H_{i}^{j}\right) \leq t_{i}$, for $j \in\left[m_{i}\right]$, where $\left(H_{i}^{1}\right)^{\kappa(H)}, \ldots,\left(H_{i}^{m_{i}}\right)^{\kappa(H)}$ are pairwise disjoint copies of $\left.H^{\kappa}(H)\right)$ in $\mathbb{Z}^{n}$.

Section 2 contains a restatement of results of [12, translating its graphcoloring context into our present one. In fact, in Theorem 3 below, a lattice 1-PTDC $\left[H_{0}, H_{1} ; m_{0}, m_{1}\right]$ is obtained; in addition, a family of lattice $\left(t_{0}, t_{1}\right)$ PTDC $\left[H_{0}, H_{1} ; m_{0}, m_{1}\right]$ 's in the graphs $\Lambda_{n}$ that extend the lattice 1-PTDC of Theorem 3 is obtained in Theorem 4.

\section{Restatement of known results}

A particular case of lattice $t$-PTDC $[H]$ is that in which $H$ is an $n$-box of $\Lambda_{n}$. For each such $H$, Theorem 2 below says that there is a lattice $n-\operatorname{PTDC}[H]$ in $\Lambda_{n}$. (In [2], $n$-boxes of unit volume in $\Lambda_{n}$ are shown to determine 1-PDDS $[H]$ 's if and only if either $n=2^{r}-1$ or $n=3^{r}-1$ ).

How to construct a lattice $n$-PTDC $[H] S$ in $\Lambda_{n}$ ? From Subsection 1.1 we know that $S$ determines a partition of $\mathbb{Z}^{n}$ into spheres $W_{n, H^{\prime}, n}$, where $H^{\prime}$ runs over the components of $[S[$. These spheres must conform a tiling associated to a lattice $L_{S} \subset \mathbb{Z}^{n}$ as follows: In each $W_{n, H^{\prime}, n}$, let $b_{1} b_{2} \cdots b_{n}$ be the vertex $a_{1} a_{2} \cdots a_{n}$ for which $a_{1}+a_{2}+\cdots+a_{n}$ is minimal. We say that this $b_{1} b_{2} \cdots b_{n}$ is the anchor of $W_{n, H^{\prime}, n}$. The anchors of the spheres $W_{n, H^{\prime}, n}$ form the lattice $L=L_{S}$. Without loss of generality we can assume that $O$ is the anchor of a $W_{n, H_{0}, n}$ whose truncated center $H_{0}$ is a component of $[S]$. Let $c_{1} c_{2} \cdots c_{n}$ be the vertex $a_{1} a_{2} \cdots a_{n}$ in $W_{n, H_{0}, n}$ for which $a_{1}+a_{2}+\cdots+a_{n}$ is maximal. Then $L_{S}$ has generating set $\left\{\left(1+c_{1}\right) e_{1},\left(1+c_{2}\right) e_{2}, \ldots,\left(1+c_{n}\right) e_{n}\right\}$ and is formed by all linear combinations of those $\left(1+c_{i}\right) e_{i},(i \in[n])$. This insures that $S$ exists and is lattice [14] (or lattice-like [1]) via $L_{S}$.

Theorem 2 [12] For each $i \in[n]$, let $P_{i}$ be a path of length $c_{i}-2$, parallel to $O e_{i}$, so $H=\Pi_{i=1}^{n} P_{i}$ is an $n$-box in $\Lambda_{n}$. Then, there is a lattice $n$-PTDC $[H]$ $S$ of $\Lambda_{n}$ with minimum $\ell_{1}$-distance 3 between the components of $[S]$.

Proof An argument for this precedes indeed the statement, and a similar proof is in 12 from a graph-coloring point of view. The claim can be proved alternatively by an additive-group epimorphism technique [1,14] modified in [12. and containing the tool used to prove Theorems 3.4

As in Theorem 2 and commented in Remark 2, Figure 1 displays a representation of the convex hulls of two induced components, in light-gray color, 
of a lattice $t$-PTDC $[H]$ in $\Lambda_{n}$, where $t=n=3$ and $H=Q_{0}^{2}$. The components of this PTDC are just 0 -cubes, the simplest case of Theorem 2 whose proof, discussed below, is contained in [12] from a graph-coloring perspective.

Theorem 3 There exists a lattice 1-PTDC $\left[Q_{2}^{2}, Q_{0}^{2} ; 2,2\right]$ (in particular a PDS) $S$ in $\Lambda_{3}$ with minimum $\ell_{1}$-distance 3 between the components of $[S]$.

As also commented in Remark 2, Theorem 3 is illustrated in Figure 1, showing in black color the components of $[S]$ in a typical FR of $L_{S}$, namely: two copies of $Q_{2}^{2}$ and two copies of $Q_{0}^{2}$, with the edges in the corresponding truncated 1-spheres shown in thick black trace; the other edges induced in the union of these 4 components are shown in thick dark-gray trace. As also commented in Remark 2, truncated 3-spheres of the 3-PTDC $\left[Q_{0}^{2}\right]$ resulting from Theorem 2 are shaded in light-gray color. Also, dark-gray color was used to indicate two other copies of $Q_{2}^{2}$ appearing in the figure that are components of $[S]$. Notice that the vertices $O, e_{1}, e_{2}, e_{3}$ are indicated in the figure.

To state Theorem 4, let $H_{0}=Q_{n-1}^{2}, H_{1}=Q_{0}^{2}, m_{0}=m_{1}=2, t_{0}=1$, $t_{1}=n-2$.

Theorem 4 There exists a lattice $(1, n-2)-\operatorname{PTDC}\left[Q_{n-1}^{2}, Q_{0}^{2} ; 2,2\right] S$ in $\Lambda_{n}$.

Remark 8 From the last observation in Remark 6, it can be deduced that the respective PTDC $S$ covers (via canonical projections) in Theorem:

- 2, an $n$-PTDC $[H]$ in a toroidal grid $\mathcal{T}=$

$$
C_{c_{1} k_{1}} \square C_{c_{2} k_{2}} \square \cdots \square C_{c_{n} k_{n}},\left(1<k_{i} \in \mathbb{Z}, \forall i \in[n]\right) ;
$$

- 3, a 1-PTDC $\left[Q_{2}^{2}, Q_{0}^{2} ; 2,2\right]$ in a cartesian product $\mathcal{T}=$ $C_{6 k_{1}} \square C_{6 k_{2}} \square C_{3 k_{3}},\left(0<k_{i}, \forall i \in[3]\right)$;

- 4. a $(1, n-2)-\operatorname{PTDC}\left[Q_{n-1}^{2}, Q_{0}^{2} ; 2,2\right]$ in a cartesian product $\mathcal{T}=$ $C_{6 k_{1}} \square \ldots \square C_{6 k_{n-1}} \square C_{3 k_{n}},\left(0<k_{i}, \forall i \in[n]\right)$.

\section{PDS's in ternary cube compounds}

Let the 2-tercube (or tersquare) $Q_{2}^{3}$ be denoted [ $\left.\emptyset\right]$, with vertices given by the 2 -tuples $x y,\left(x, y \in F_{3}=\{0,1,2\}\right)$. As a graph, $[\emptyset]=(00,10,20) \square(00,01,02)$, the cartesian product of two triangles whose vertex sets are both $F_{3}$. The 1-sub-tercubes $Q_{1}^{3}$ of the 2-tercube $[\emptyset]$ have vertex sets $\{0 y\}_{y \in F_{3}},\{1 y\}_{y \in F_{3}}$, $\{2 y\}_{y \in F_{3}},\{x 0\}_{x \in F_{3}},\{x 1\}_{x \in F_{3}},\{x 2\}_{x \in F_{3}}$. They will be indicated $x^{0}, x^{1}, x^{2}$, $y^{0}, y^{1}, y^{2}$, respectively. To each of these triangles $t^{s}\left(t \in\{x, y\} ; s \in F_{3}\right)$ of $[\emptyset]$ we glue, or lock, a corresponding tersquare $\left[\begin{array}{l}t \\ s\end{array}\right]$ intersecting $[\emptyset]$ exactly in $t^{s}$.

This way, six tersquares $\left[\begin{array}{l}x \\ 0\end{array}\right], \ldots,\left[\begin{array}{l}y \\ 2\end{array}\right]$ are obtained (that we call subcentral tersquares) that intersect $[\emptyset]$ respectively in the triangles $x^{0}, \ldots, y^{2}$. Next, we glue, or lock, to the remaining new triangles (i.e., other than those already in $[\emptyset])$, a set of nine new tersquares that we denote $\left[\begin{array}{l}x y \\ 00\end{array}\right], \ldots,\left[\begin{array}{l}x y \\ 22\end{array}\right]$ and call corner tersquares. These nine tersquares share merely a vertex with $[\emptyset]$. The resulting graph $[[\emptyset]]$, that will be referred to as a 2 -hive, contains a total of $1+6+9=16$ tersquares, where the central tersquare $[\emptyset]$ of $[[\emptyset]]$ shares just one triangle with 


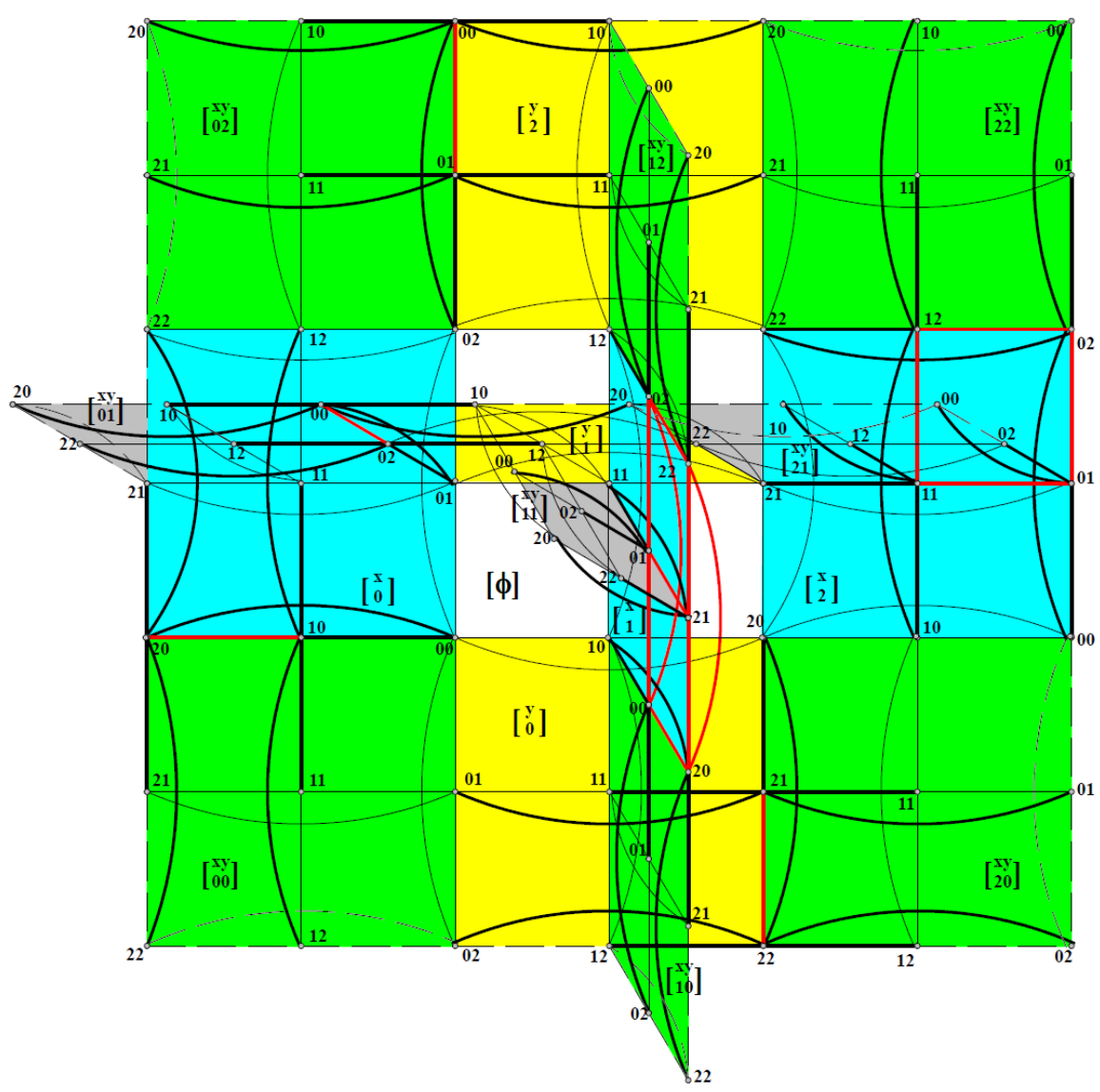

Fig. 3 Representing a PDS of [[Ø]] in $\Gamma_{2}$

The dominating edges are drawn in thick dark trace, allowing the reader to verify that all vertices of [[Ø]] not in $S$ are dominated as indicated in Remark 1. (It is elementary to verify that no isolated PDS in $[[\emptyset]]$ exists). The remaining edges of $[[\emptyset]]$, not in thick (either ' or black) trace, are in dashed black trace if they are "exterior" edges of [[Ø]] (i.e., those shared by 2-hives other than [[Ø]] in $\left.\Gamma_{2}\right)$ and in thin black trace otherwise (i.e., if they are "interior" edges of [[Ø]]). Motivation for having pursued such a non-isolated PDS arose as a palliative remedy for the impossibility of having here a PDS like the Livingston-Stout PDS in the grid $P_{4} \square P_{4}$ [17, which happens to be the only isolated PDS in grid graphs $P_{m} \square P_{n}$, for $2<\min \{m, n\}[6$.

Theorem 5 There exists a non-isolated PDS, or 1-PTDC[Q $Q_{1}^{2}, Q_{2}^{2}, Q_{1}^{3} \square Q_{1}^{2} ; 4$, $1,1]$, in the 2-hive $[[\emptyset]] \subset \Gamma_{2}$.

Proof The claimed PDS in [[Ø]] is formed by the vertex sets of the following components (of its induced subgraphs): 


\begin{tabular}{|c|c|c|c|c|c|c|c|c|c|}
\hline$\left[\begin{array}{l}x x y y \\
02121\end{array}\right]$ & {$\left[\begin{array}{l}x x x y \\
0212\end{array}\right]$} & {$\left[\begin{array}{l}20 \\
x x y y \\
0121\end{array}\right]$} & {$\left[\begin{array}{l}x x y \\
012\end{array}\right]$} & $\begin{array}{l}00 \\
{\left[\begin{array}{l}x y y \\
121\end{array}\right]}\end{array}$ & {$\left[\begin{array}{l}\mathrm{xy} \\
12\end{array}\right]$} & $\begin{array}{l}20 \\
\mathrm{xxyy} \\
2121\end{array}$ & {$\left[\begin{array}{l}\mathrm{xxy} \\
212\end{array}\right]$} & $\begin{array}{l}00 \\
5 x x y y \\
20121\end{array}$ & {$\left[\begin{array}{l}\mathrm{xxxy} \\
2012\end{array}\right]$} \\
\hline$\left[\begin{array}{l}\mathrm{xxyy} \\
0221\end{array}\right]$ & {$\left[\begin{array}{l}\mathrm{xxy} \\
022\end{array}\right]$} & {$\left[\begin{array}{l}\mathrm{xyy} \\
021\end{array}\right]$} & {$\left[\begin{array}{l}\mathrm{xy} \\
02\end{array}\right]$} & {$\left[\begin{array}{l}\mathrm{yy} \\
21\end{array}\right]$} & {$\left[\begin{array}{l}\mathrm{y} \\
2\end{array}\right]$} & {$\left[\begin{array}{l}\mathrm{xyy} \\
221\end{array}\right]$} & {$\left[\begin{array}{l}\mathrm{xy} \\
22\end{array}\right]$} & {$\left[\begin{array}{l}\mathrm{xxyy} \\
2021\end{array}\right]$} & {$\left[\begin{array}{l}\mathrm{xxy} \\
202\end{array}\right]$} \\
\hline$\left[\begin{array}{l}\mathrm{xxxy} \\
0211\end{array}\right.$ & {$\left[\begin{array}{l}\mathrm{xxx} \\
021\end{array}\right]$} & $\begin{array}{r}22 \\
{\left[\begin{array}{l}x x y \\
011\end{array}\right]}\end{array}$ & {$\left[\begin{array}{l}\mathrm{xx} \\
01\end{array}\right]$} & $\begin{array}{ll}02 & \\
{\left[\begin{array}{l}\mathrm{xy} \\
11\end{array}\right]}\end{array}$ & & $22\left[\begin{array}{l}\mathrm{xxy} \\
211\end{array}\right]$ & {$\left[\begin{array}{l}x x \\
21\end{array}\right]$} & {$\left[\begin{array}{l}02 \\
{\left[\begin{array}{l}x x y \\
2011\end{array}\right]}\end{array}\right]$} & {$\left[\begin{array}{l}\mathrm{xxx} \\
201\end{array}\right]$} \\
\hline$\left[\begin{array}{l}\mathrm{xxy} \\
021\end{array}\right]$ & {$\left[\begin{array}{l}\mathrm{xx} \\
02\end{array}\right]$} & {$\left[\begin{array}{l}x y \\
01\end{array}\right]$} & {$\left[\begin{array}{l}x \\
0\end{array}\right]$} & {$\left[\begin{array}{l}y \\
1\end{array}\right]$} & {$[\phi$} & {$\left[\begin{array}{l}\mathrm{xy} \\
21\end{array}\right]$} & {$\left[\begin{array}{l}x \\
2\end{array}\right]$} & {$\left[\begin{array}{l}x \mathrm{xy} \\
201\end{array}\right]$} & {$\left[\begin{array}{l}\mathrm{xx} \\
20\end{array}\right]$} \\
\hline$\left[\begin{array}{l}\mathrm{xxyyy} \\
02101\end{array}\right.$ & {$\left[\begin{array}{l}\mathrm{xxxy} \\
0210\end{array}\right]$} & {$\left[\begin{array}{l}20 \\
0101\end{array}\right]$} & {$\left[\begin{array}{l}\mathrm{xxy} \\
010\end{array}\right]$} & $\begin{array}{r}00 \\
{\left[\begin{array}{l}\mathrm{xyy} \\
101\end{array}\right]}\end{array}$ & {$\left[\begin{array}{l}x y \\
10\end{array}\right]$} & $\left.\begin{array}{l}20 \\
\mathrm{xxyy} \\
2101\end{array}\right]$ & {$\left[\begin{array}{l}\mathrm{xxy} \\
210\end{array}\right]$} & $\begin{array}{l}00 \\
\text { xxxyy } \\
20101\end{array}$ & {$\left[\begin{array}{l}\mathrm{xxxy} \\
2010\end{array}\right]$} \\
\hline$\left[\begin{array}{l}\mathrm{xxyy} \\
0201\end{array}\right]$ & {$\left[\begin{array}{l}\mathrm{xxy} \\
020\end{array}\right]$} & {$\left[\begin{array}{l}\mathrm{xyy} \\
001\end{array}\right]$} & {$\left[\begin{array}{l}\mathrm{xy} \\
00\end{array}\right]$} & {$\left[\begin{array}{l}\mathrm{yy} \\
01\end{array}\right]$} & {$\left[\begin{array}{l}\mathrm{y} \\
0\end{array}\right]$} & {$\left[\begin{array}{l}\mathrm{xyy} \\
201\end{array}\right]$} & {$\left[\begin{array}{l}\mathrm{xy} \\
20\end{array}\right]$} & {$\left[\begin{array}{l}\mathrm{XXY} \\
2001\end{array}\right]$} & {$\left[\begin{array}{l}x x y \\
200\end{array}\right]$} \\
\hline$\left[\begin{array}{l}x x x y y y \\
021021\end{array}\right]$ & {$\left[\begin{array}{l}x x x y y \\
02102\end{array}\right]$} & {$\left[\begin{array}{l}22 \\
x x y y y \\
01200\end{array}\right]$} & {$\left[\begin{array}{l}x x y y \\
0120\end{array}\right]$} & $\left.\begin{array}{c}02 \\
\text { xyyy } \\
1021\end{array}\right]$ & {$\left[\begin{array}{l}\mathrm{xyy} \\
102\end{array}\right]$} & $\begin{array}{l}22 \\
x x y y y \\
21021\end{array}$ & {$\left[\begin{array}{l}x y y y \\
2012\end{array}\right]$} & $\begin{array}{l}\mathbf{0 2} \\
\mathrm{xxxyyy} \\
201021\end{array}$ & {$\left[\begin{array}{l}\mathrm{xxxyy} \\
20102\end{array}\right.$} \\
\hline$\left[\begin{array}{l}\text { xyyyy } \\
02021\end{array}\right]$ & {$\left[\begin{array}{l}x x y y \\
0202\end{array}\right]$} & {$\left[\begin{array}{l}\text { xyyy } \\
0021\end{array}\right]$} & {$\left[\begin{array}{l}x y y \\
002\end{array}\right]$} & {$\left[\begin{array}{l}\mathrm{yyy} \\
021\end{array}\right]$} & {$\left[\begin{array}{l}\mathrm{yy} \\
02\end{array}\right]$} & {$\left[\begin{array}{l}\text { xxyy } \\
2102\end{array}\right]$} & {$\left[\begin{array}{l}x y y \\
202\end{array}\right]$} & {$\left[\begin{array}{l}\mathrm{xxyyy} \\
20021\end{array}\right]$} & {$\left[\begin{array}{l}x x y y \\
2002\end{array}\right]$} \\
\hline
\end{tabular}

Fig. 4 Planar projection of a portion of $\Gamma_{2}$ larger than in Figure 3

(a) the edge $(00,01)$ of triangle $(00,01,02)$ shared by tersquares $\left[\begin{array}{l}y \\ 2\end{array}\right]$ and $\left[\begin{array}{l}x y \\ 02\end{array}\right]$;

(b) the edge $(10,20)$ of triangle $(00,10,20)$ shared by tersquares $\left[\begin{array}{l}x \\ 0\end{array}\right]$ and $\left[\begin{array}{l}x y \\ 00\end{array}\right]$;

(c) the edge $(21,22)$ of triangle $(20,21,22)$ shared by tersquares $\left[\begin{array}{l}y \\ 0\end{array}\right]$ and $\left[\begin{array}{c}x y \\ 20\end{array}\right]$;

(d) the edge $(00,02)$ of triangle $(00,02,01)$ shared by tersquares $\left[\begin{array}{l}y \\ 1\end{array}\right]$ and $\left[\begin{array}{l}x y \\ 01\end{array}\right]$;

(e) the 4 -cycle $(02,12,11,01)$ of tersquare $\left[\begin{array}{l}x \\ 2\end{array}\right]$, where its edge $(02,12)$ (of triangle $\Delta=(02,12,22)$ ) is shared (with $\Delta$ ) with tersquare $\left[\begin{array}{l}x y \\ 22\end{array}\right]$;

(f) the triangular prism in $\left[\begin{array}{l}x \\ 1\end{array}\right]$ formed by triangles $(00,01,02)$ and $(20,21,22)$ and edges $(00,20),(02,22)$ (of triangle $(02,22,12)$, shared by tersquare $\left.\left[\begin{array}{c}x y \\ 22\end{array}\right]\right)$ and $(01,21)$ (of triangle $(01,21,11)$, shared by tersquare $\left.\left[\begin{array}{l}x y \\ 11\end{array}\right]\right)$.

As can be verified in Figure 3, the edges departing from these red-edge components cover all other vertices of $[[\emptyset]]$, proving the theorem.

Figure 3 may be augmented by adding the tersquares $\left[\begin{array}{ll}x x & y \\ i & j\end{array}\right]$, with $i \neq j$ in $F_{3}$, etc. One may continue by adding tersquares $\left[\begin{array}{c}x x \cdots x x y y \cdots y y \\ i j \ldots k l m n \cdots p\end{array}\right]$ with $i \neq j \neq$ $\ldots \neq k \neq l$ (resp. $m \neq n \neq \ldots \neq p \neq q$ ), i.e. not two contiguous equal values under the $x$ 's (resp. $y$ 's). Taking $i, j, \ldots, k, l, m, n, \ldots p, q$ in $F_{3}$ to be contiguously different with just two values, (e.g. 0,1 ; resp. 0,2 ; resp. 1,2 , in the upper-left; resp. upper-right; resp. lower-left quarter in Table I) is a way of obtaining 3 sub-lattices of $\Gamma_{2}$, each isomorphic to $\Lambda_{2}$, as in Table I. But there is an infinite family of "parallel" sub-lattices in $\Gamma_{2}$ for each of the 9 
sublattice types, one per each 4-cycle of a typical tersquare as in the lower-left quarter of Table I. For example, the type with values 0,2 represents "parallel" sub-lattices having "shortest" tersquares $[\emptyset],\left[\begin{array}{l}x \\ 1\end{array}\right],\left[\begin{array}{l}y \\ 1\end{array}\right],\left[\begin{array}{l}x x \\ 01\end{array}\right],\left[\begin{array}{l}y y \\ 01\end{array}\right],\left[\begin{array}{l}x x \\ 21\end{array}\right],\left[\begin{array}{c}y y \\ 21\end{array}\right]$, etc.,

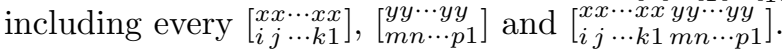

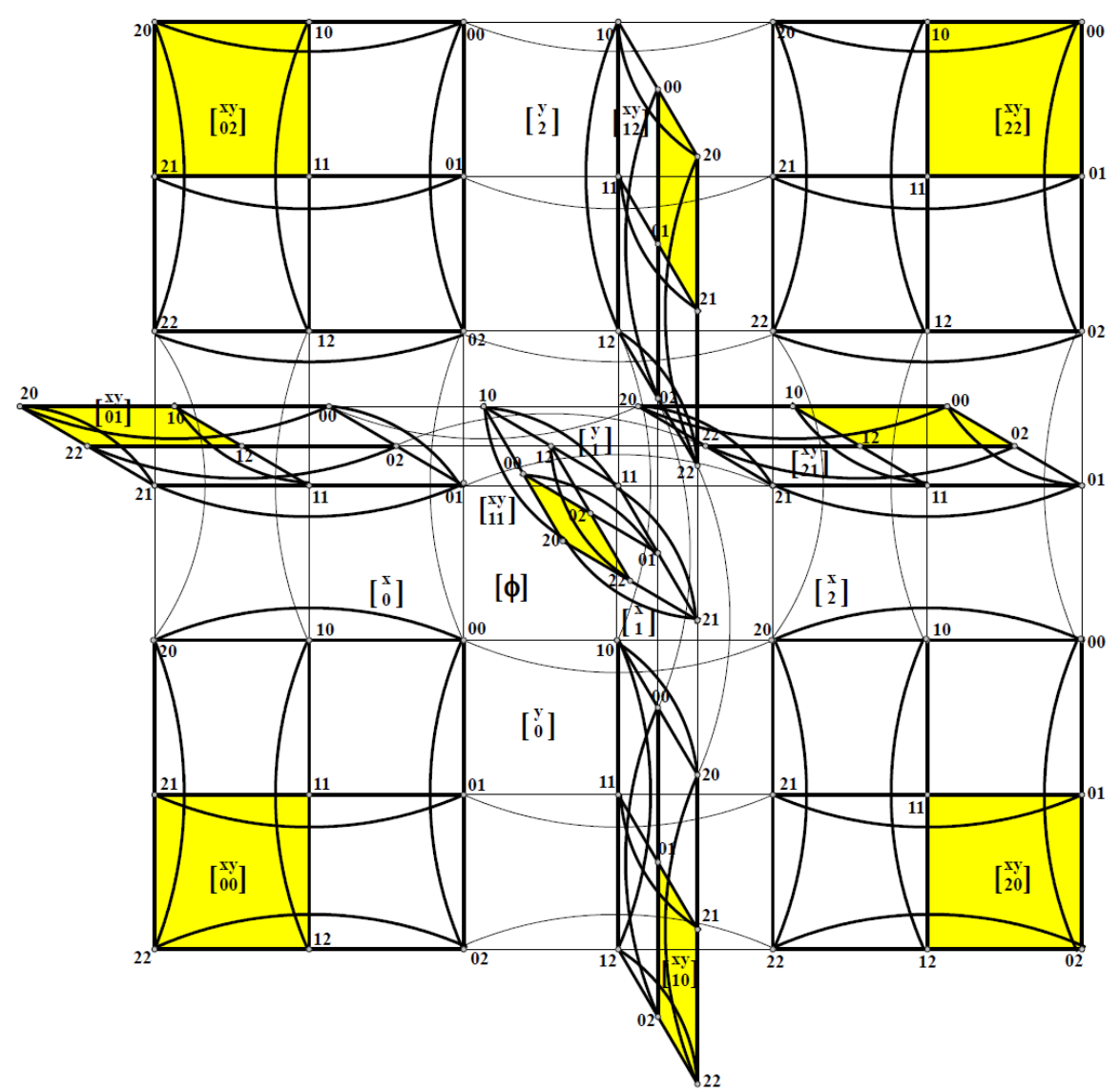

Fig. 5 Components of a $2-\operatorname{PTDC}\left[Q_{2}^{2}\right]$ of $[[\emptyset]]$ in $\Gamma_{2}$

These sub-lattices can be refined by replacing each edge $e$ in them by the 2-path closing a triangle with $e$ and adding more vertices and additional edges out of them to the new vertices obtained by the said replacement, in order to distinguish the glued or locked 2-tercubes in $\Gamma_{2}$. In particular, Figure 3 is obtained from such a procedure by starting from a plane containing the upperright quarter of Table I. Figure 4 shows a projection of a partial extension of Figure 3 in such a plane, where tersquare colors are kept as in Figure 3 , including if they are projected into a 2-path. Colors here still are lightblue, blue, green and light-gray. Each tersquare in Figure 4, represented by four squares with the common central vertex 11, is just recognizable by its 
upper-left vertex denomination presence. Of the four such squares, the lowerright one has the corresponding tersquare denomination. The other tersquare denominations in the three remaining squares correspond to the counterpart denominations in the representation of $[\emptyset]$.

Similarly, a graph $\Gamma_{n}$ may be defined as a compound of glued $n$-tercubes along their codimension 1 ternary cubes in a likewise manner, for any $2<$ $n \in \mathbb{Z}$. Such a compound may be referred to as a ternary $n$-cube compound. The $n$-tuples representing the vertices of such $\Gamma_{n}$ are given in terms of the $n$ tercubes […...] containing them; these $n$-tuples are assigned locally by reflection on the $(n-1)$-subtercubes, as done in Figure 3 for $n=2$, which represents the 2-hive $[[\emptyset]]$ with $[\emptyset]$ at its center, and its neighboring tersquares $\left[\begin{array}{l}x \\ 0\end{array}\right],\left[\begin{array}{l}x \\ 1\end{array}\right],\left[\begin{array}{l}x \\ 2\end{array}\right]$ in light-blue background, $\left[\begin{array}{l}y \\ 0\end{array}\right]$, and $\left[\begin{array}{l}y \\ 1\end{array}\right],\left[\begin{array}{l}y \\ 2\end{array}\right]$ in yellow background, and the tersquares neighboring those tersquares, namely $\left[\begin{array}{c}x y \\ i j\end{array}\right],\left(i, j \in F_{3}\right)$, in green and light-gray backgrounds. It can be seen that $\Gamma_{n}$ may be considered as a superset of $\Lambda_{n}$ in three different ways, as was commented above in relation to Table I for $n=2$.

We pose the following.

Question 2 Does there exist a non-isolated PDS $S$ in $\Gamma_{n}$, for $n \geq 2$ ? If so, could $S$ behave like a lattice, for example by restricting itself to a lattice PDS over any sub-lattice of $\Gamma_{n}$, as exemplified in Table I?

\section{Isolated 2-PTDC's in the ternary square compound $\Gamma_{2}$}

In this section, we keep working in $\Gamma_{2}$, but slightly modifying the definition of a $\kappa$-PTDC by replacing the used Hamming distance $H$ by the graph distance distance of $\Gamma_{2}$. After all, it is clear that the Hamming distance in our graphtheoretical context is nothing else than the graph distance. Then, we have the following result.

Theorem 6 There exists 262144 isolated 2-PTDC's in the 2-hive $[[\emptyset]] \subset \Gamma_{2}$.

Proof In Figure 5, the nine copies $\left[{ }_{i j}^{x y}\right],\left(i, j \in F_{3}\right)$, of the tersquare are shown with its edges in thick black trace, against the thin black trace of the remaining edges of the 2-hive [[Ø]]. These nine copies happen to be the truncated 2spheres centered at the vertices of an isolated PTDC of [[Ø]] constituted as a selection of one vertex per yellow-faced 4-cycle in the figure. We may say that these yellow-faced 4-cycles are the "external" 4-cycles of $[[\emptyset]]$. So, there are $4^{9}=2621442$-PTDC's in $\Gamma_{2}$.

Corollary 1 There exists a 2-PTDC[Q 2$]$ in the 2-hive $[[\emptyset]]$.

Proof The yellow-faced 4-cycles are copies of $Q_{2}^{2}$ and induce the components of the claimed 2-PTDC- $\left[Q_{2}^{2}\right]$.

Theorem 7 There exists a 2-PTDC[Q $\left.Q_{2}^{2}\right]$ in $\Gamma_{2}$, as well as an infinite number of isolated 2-PTDC in $\Gamma_{2}$. 


\section{TABLE II}

\begin{tabular}{|c|c|c|c|c|c|c|c|}
\hline$[x$ & $\int^{x y}$ & {$[x y$} & {$[x y$} & {$[x]$} & {$[x y$} & {$[x y$} & ryy \\
\hline$[0$ & Loo & Lo1 & 02 & {$[0$} & 00 & 01 & 02 \\
\hline$[x x$ & $x x y$ & {$[x x y$} & {$[x x y$} & {$[x x]$} & {$[x x y$} & {$[x x y$} & {$[x x y$} \\
\hline โ01. & L010 & L011 & L012 & โ02 & L020 & $\lcm{021}$ & l022 \\
\hline$[x x x$ & {$[x x x y$} & {$[x x x y$} & {$[x x x y$} & $\lceil x x x$ & {$[x x y$} & $x x x y$ & {$[x x x y$} \\
\hline 010 & 0100 & 0101 & 0102 & {$\left[\begin{array}{l}x \\
020\end{array}\right.$} & 0200 & 0201 & 0202 \\
\hline & {$[x x x y$} & {$[x x x y$} & {$[x x x y$} & {$[x x x$} & {$[x x x y$} & {$[x x x y$} & {$[x x x y$} \\
\hline$[012$ & L0120 & 0121 & L0122 & L021 & L0210 & L0211 & L0212 \\
\hline$\Gamma^{y}$ & ${ }^{x} x y$ & $\int^{x y}$ & $\bar{f} x y$ & $\Gamma^{y}$ & $\overline{x y}$ & {$[x y$} & {$[x y$} \\
\hline$\lfloor 0$ & 100 & $\lfloor 10$ & $\lfloor 20$ & Lo & L00 & 10 & {$[20$} \\
\hline$\left[\begin{array}{l}y y \\
01\end{array}\right.$ & {$\left[\begin{array}{c}x y y \\
001\end{array}\right.$} & {$[x y y$} & {$\left[\begin{array}{ll}x y y \\
001\end{array}\right.$} & {$\left[\begin{array}{l}y y \\
0 ?\end{array}\right.$} & {$\left[\begin{array}{c}x y y \\
0\end{array}\right.$} & {$[x y y$} & {$[x y y$} \\
\hline Гyyy & гxyy & гxyy & гxyy & Гу⿰у丨 & гxyy & гxyyy & гxyy \\
\hline 010 & 0010 & L1010 & L1010 & 020 & [0020 & 1020 & 1020 \\
\hline [yyy & [xyyy & [xyyy & [xyyy & Гyyy & гxyyy & xyyy & гxyy \\
\hline$[012$ & {$[0012$} & L1012 & {$[1012$} & {$[012$} & 0021 & 1021 & 1021 \\
\hline$[x$ & $\Gamma^{x y}$ & $\Gamma^{x y}$ & $\Gamma^{x y}$ & {$[x$} & $\Gamma^{x y}$ & {$[x y$} & {$[x y$} \\
\hline$\left[\begin{array}{l}1 \\
\text {. }\end{array}\right.$ & 10 & {$[11$} & $\lfloor 12$ & {$\left[\begin{array}{l}1 \\
1\end{array}\right.$} & 10 & L11 & 12 \\
\hline$\lceil x$ & {$[x x y$} & {$[x x y$} & {$\left[\begin{array}{ll}x y y \\
\text {. }\end{array}\right.$} & & [xyy & [xy & {$\left[\begin{array}{ll}x x y \\
0\end{array}\right.$} \\
\hline$\left[\begin{array}{l}10 \\
10\end{array}\right.$ & L100 & โ101 & 102 & {$\left[\begin{array}{l}\infty \\
12\end{array}\right.$} & $\lfloor 120$ & โ121 & โ122 \\
\hline$[x x x$ & {$[x x x y$} & {$[x x y y$} & {$[x x x y$} & $\lceil x x x$ & {$[x x y$} & {$[x x x y$} & {$[x x x y$} \\
\hline [101 & {$[1010$} & {$[1011$} & {$[1012$} & L120 & {$[1200$} & $\lfloor 1201$ & $\lfloor 1202$ \\
\hline$\left[\begin{array}{l}x x x \\
102\end{array}\right.$ & {$\left[\begin{array}{l}x x x y \\
1020\end{array}\right.$} & {$\left[\begin{array}{l}x x x y \\
102 y\end{array}\right.$} & {$\left[\begin{array}{l}x x x y \\
1022\end{array}\right.$} & {$\left[\begin{array}{ll}x x & x\end{array}\right.$} & {$\left[\begin{array}{cc}x x y \\
1\end{array}\right.$} & {$\left[\begin{array}{l}x x y \\
1\end{array}\right.$} & {$\left[\begin{array}{l}x x y \\
121\end{array}\right.$} \\
\hline$\Gamma^{y}$ & $\lceil x y$ & $\Gamma x y$ & $\lceil x y$ & $\Gamma y$ & $\ulcorner x y$ & $5 x y$ & $5 x y$ \\
\hline 1 & 01 & 11 & 21 & 1 & 01 & 11 & 21 \\
\hline$\lceil y y$ & гxy & Гxy & гxyy & 「yy & [xyy & rxyy & гxyy \\
\hline$[10$ & L010 & [110 & [210 & {$[12$} & โ012 & โ112 & {$[212$} \\
\hline 5yy & [xyyy & гxyyy & [xyy & ᄃyy & [xyyy & [xyyy & [xyyy \\
\hline$\left[\begin{array}{l}0 \\
101\end{array}\right.$ & 0101 & 1101 & 2101 & lo & 0120 & 1120 & 2120 \\
\hline [yyy & [xyyy & זxyy & 「xyyy & [yyy & гxyyy & [xyyy & [xyyy \\
\hline$[102$ & $\lfloor 0102$ & $\lfloor 1102$ & 2102 & L121 & $\lfloor 0121$ & L1121 & 2121 \\
\hline 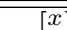 & $=[x y$ & $=[x y$ & $=[x y$ & $\bar{c}\lceil x$ & $=[x y$ & $=\lceil x y$ & $=[x y$ \\
\hline$\lfloor 2$ & 20 & $\lfloor 21$ & $\lfloor 22$ & {$[2$} & 20 & {$[21$} & {$[22$} \\
\hline$\left[\begin{array}{l}x x \\
20\end{array}\right.$ & {$\left[\begin{array}{c}x x y \\
200\end{array}\right.$} & {$\left[\begin{array}{c}x x y \\
0\end{array}\right.$} & {$\left[\begin{array}{c}x x y \\
202\end{array}\right.$} & {$\left[\begin{array}{l}x x \\
21\end{array}\right.$} & {$\left[\begin{array}{l}x x y \\
0\end{array}\right.$} & {$[x x y$} & {$[x x y$} \\
\hline$\lceil x x x$ & $x x x y$ & гxxy & 「xxxy & 「xx & Гxxxy & $x x x y$ & {$\left[\begin{array}{l}{[212} \\
x\end{array}\right.$} \\
\hline 201 & 2010 & 2011 & L2012 & 210 & 2100 & L2101 & [2102 \\
\hline$[x \times x$ & {$[x x x y$} & {$[x x x y$} & {$[x x x y$} & $\lceil x x x$ & {$[x x x y$} & {$[x x x y$} & {$[x x x y$} \\
\hline 202 & 2020 & 2021 & {$[2022$} & 212 & 2120 & 2121 & {$[2122$} \\
\hline$[y$ & $x^{x y}$ & $\Gamma^{x y}$ & {$[x y$} & $y$ & $\int^{x y}$ & {$[x y$} & $\Gamma^{x y}$ \\
\hline$[2$ & L02 & $\lfloor 12$ & {$[22$} & {$[2$} & L02 & L12 & {$[22$} \\
\hline & r xyy & {$\left[\begin{array}{cc}x y y \\
\text {. }\end{array}\right.$} & {$[x y y$} & [yy & {$[x y y$} & ryy & [xyy \\
\hline$[20$ & 020 & 120 & 220 & 21 & {$[021$} & L121 & 221 \\
\hline [yyy & гxyy & гxyy & гxyy & Гyy & гxyy & xyyy & гxyy \\
\hline$[201$ & [0201 & {$[1201$} & {$[2201$} & {$[210$} & L0210 & โ1210 & 2210 \\
\hline [yyy & [xyyy & 「хуyy & 「хуyy & [yyy & Гxyyy & [xyyy & [xyyy \\
\hline$[202$ & {$[0202$} & $\lfloor 1202$ & {$[2202$} & {$[212$} & 0212 & {$[1212$} & {$[2212$} \\
\hline
\end{tabular}

Proof We consider the following 18 triple unions of tercubes in the 2-hive [[Ø]]:

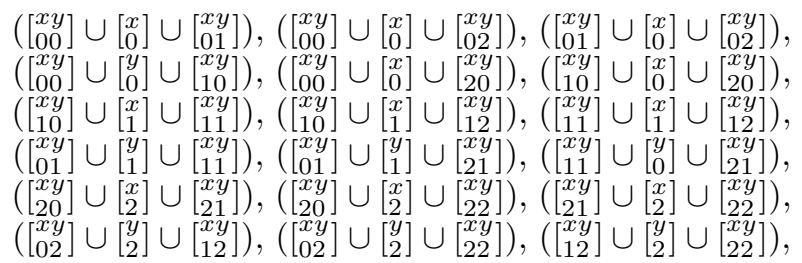

where the union of the three graphs in each line will be called a quarter of $[[\emptyset]]$, as well as six pairs of new 2-hives overlapping [[Ø]] in the six shown quarters, having these pairs of new 2-hives the following respective pairs of central tersquares:

$$
\left(\left[\begin{array}{l}
x x \\
01
\end{array}\right],\left[\begin{array}{l}
x x \\
02
\end{array}\right]\right),\left(\left[\begin{array}{l}
y y \\
01
\end{array}\right],\left[\begin{array}{l}
y y \\
02
\end{array}\right]\right),\left(\left[\begin{array}{l}
x x \\
10
\end{array}\right],\left[\begin{array}{l}
x x \\
12
\end{array}\right]\right),\left(\left[\begin{array}{l}
y y \\
10
\end{array}\right],\left[\begin{array}{l}
y y \\
12
\end{array}\right]\right),\left(\left[\begin{array}{l}
x x \\
20
\end{array}\right],\left[\begin{array}{l}
x x \\
21
\end{array}\right]\right),\left(\left[\begin{array}{l}
y y \\
20
\end{array}\right],\left[\begin{array}{l}
y y \\
21
\end{array}\right]\right) .
$$

These six pairs account for 12 2-hives, each having 3 corner tersquares in common with $[[\emptyset]]$ separated by a common subcentral tersquare; the remaining 6 corner tersquares in each of the said 12 2-hives contain each a yellow-faced 
4-cycles; in each such yellow-faced 4-cycle, we can select a vertex so that each involved 2-hive has an isolated 2-PTDC formed by such vertices; the union of all the isolated 2-PTDC's so obtained constitutes an isolated 2-PTDC of the union of all those 2-hives while the vertices of all yellow-faced 4-cycles constitute a 2 -PTDC $\left[Q_{2}^{2}\right]$.

Let us check the 12 2-hives mentioned above, ordered in the form they were already presented. They show up in Table II, where each such 2-hive [[U]] with

$$
[U] \in\left\{\left[\begin{array}{l}
x x \\
01
\end{array}\right],\left[\begin{array}{l}
x x \\
02
\end{array}\right],\left[\begin{array}{l}
y y \\
01
\end{array}\right],\left[\begin{array}{l}
y y \\
02
\end{array}\right],\left[\begin{array}{l}
x x \\
10
\end{array}\right],\left[\begin{array}{l}
x x \\
12
\end{array}\right],\left[\begin{array}{l}
y y \\
10
\end{array}\right],\left[\begin{array}{l}
y y \\
12
\end{array}\right],\left[\begin{array}{l}
x x \\
20
\end{array}\right],\left[\begin{array}{l}
x x \\
21
\end{array}\right],\left[\begin{array}{l}
y y \\
20
\end{array}\right],\left[\begin{array}{l}
y y \\
21
\end{array}\right]\right\}
$$

is given as a corresponding $4 \times 4$-array of tersquares. In such a $4 \times 4$-array, the tersquares cited in the first row are shared with the 2-hive [[Ø]]; the central tersquare $[U]$ is given as the first entry of the second row; the subcentral tersquares are given as the six remaining entries of the second row and the first column, and the corner tersquares that are not in $[[\emptyset]]$ are the six remaining entries, in the third and fourth rows. In all, there are 72 such corner tersquares, that added to the 9 of $[[\emptyset]]$ yields 81 corner tersquares in the 12 2-hives of Table II. It is clear that this 81 corner tersquares are distinct and separated, so that by selecting a vertex in each of their corresponding yellow-faced 4-cycles, the claimed 2-PTDC is initiated. To continue iteratively, starting with the upperleft 2-hive $\left.\left[\begin{array}{c}x x \\ 00\end{array}\right]\right]$ and ending with the lower-right 2-hive $\left[\left[\begin{array}{c}y y \\ 21\end{array}\right]\right]$, both in Table II, we have the following (vertically disposed) correspondences from their new subcentral tersquares (other than $\left[x_{0}\right]$ and $\left[y_{2}\right]$ ) to the new pairs of 2-hives to be considered in the iteration for considerarion of new yellow-faced 4-cycles and candidates for vertices in PDS's:

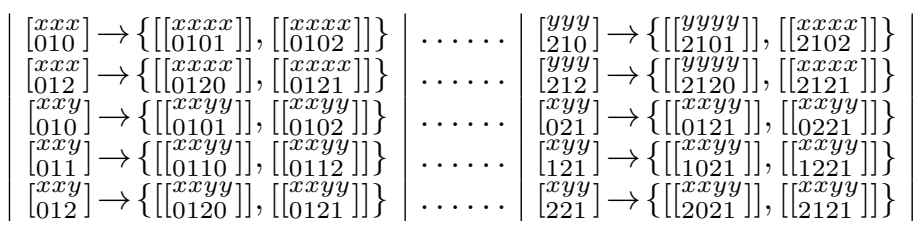

and so on. In (1), each target pair in the last 3 lines has each of its two 2-hives arriving from two different source tersquares; for example, $\left.\left[\begin{array}{l}x x y y \\ 0121\end{array}\right]\right]$ is in the pair $\left.\left\{\left[\begin{array}{c}x x y y \\ 0120\end{array}\right]\right],\left[\left[\begin{array}{c}x x y y \\ 0121\end{array}\right]\right]\right\}$ in the fifth row on the left of (1) as the target of the tersquare $\left[\begin{array}{c}x x y \\ 012\end{array}\right]$ and also in the pair $\left\{\left[\left[\begin{array}{l}x x y y \\ 0121\end{array}\right]\right],\left[\left[\begin{array}{c}x x y y \\ 0221\end{array}\right]\right]\right\}$ in the third row on the right of $(1)$ as the targer of the tersquare $\left[\begin{array}{l}x y y \\ 021\end{array}\right]$. We observe that in this iterative process of reconstructing $\Gamma_{2}$ via overlapping of 2-hives away from [[Ø]], the central tersquares of participating 2-hives are those of the form $\left[\begin{array}{c}x \cdots x y \cdots y \\ \cdots \cdots \cdots \cdots\end{array}\right]$, where the number of $x$ 's and the number of $y$ 's are both even.

By iteration, we obtain both a $2-\mathrm{PTDC}\left[Q_{2}^{2}\right]$ formed by the vertices of all yellow-faced 4-cycles in successive corner tersquares and an infinite number of isolated 2-PTDC's of $\Gamma_{2},(262144$ per 2-hive).

Conjecture 2 There exists an isolated $n$-PTDC in $\Gamma_{n}, \forall n>2$.

Question 3 Does there exist a suitable way of declaring a susbset $S \subset V\left(\Gamma_{n}\right)$ to be periodic, or periodic-like, that extends the notion of periodicity of $\Lambda_{n}$ 
at the end of Remark 6 to one in $\Gamma_{n}$ ? so that a replacement of the notion of "quotient" or "toroidal" graphs of $\Lambda_{n}$ can be found that way for $\Gamma_{n}$ ?

\section{References}

1. C. Araujo, I. Dejter and P. Horak, A generalization of Lee codes, Designs, Codes and Cryptography, 70 (2014), 77-90.

2. S. Buzaglo and T. Etzion, Tilings by $(0.5, n)$-Crosses and Perfect Codes., SIAM Jour. Discrete Math., 27 (2013), 1067-1081.

3. R. Calderbank, ed., Different aspects of coding theory: AMS Short Course, San Francisco, CA, AMS, Proc. Symp. Appl. Math., 501995.

4. A. Campello, G. C. Jorge, J. E. Strapasson, S. I. R. Costa, Perfect codes in the $\ell_{p}$ distance, European Journal of Combinatorics, 53 (2016),72-85.

5. G. Cohen, I. Honkala, S. Litsyn and A. Lobstein, Covering codes, Elsevier 1997.

6. I. Dejter, A. Delgado, Perfect dominating sets in rectangular grid graphs, J. Combin. Math. Combin. Comput., 70 (2009), 177-196.

7. I. Dejter, L. Fuentes and C. Araujo, There is but one PDS in $\mathbb{Z}^{3}$ inducing just square components, Bull. Inst. Combin. Appl., 82 (2018), 30-41.

8. I. Dejter and O. Serra, Efficient dominating sets in Cayley graphs, Discrete Applied Mathematics, 129 (2003), 319-328.

9. I. Dejter and P. Weichsel, Twisted perfect dominating subgraphs of hypercubes, Congr. Numer. 94 (1993), 67-78.

10. T. Etzion, Product constructions for perfect Lee codes, IEEE Transactions in Information Theory, 57 (2011), 7473-7481.

11. T. Etzion, Tilings with generalized Lee spheres, in: J.-S. No et al., eds., Mathematical Properties of Sequences, Springer 726 (2003), 181-198.

12. L. Fuentes, I. Dejter and C. Araujo, Rainbow perfect domination in lattice graphs, Electr. Jour. Graph Th. and Appl., 6 (1) (2018), 95-112

13. S. Golomb and K. Welch, Perfect codes in the Lee distance and the packing of polyominoes, SIAM Jour. Applied Math., 18 (1970), 302-317.

14. P. Horak and B. F. AlBdaiwi, Diameter perfect Lee codes, IEEE Transactions of Information Theory, 58 (2012),5490-5499.

15. P. Horak and O. Grosek, A new approach towards the Golomb-Welch conjecture. European Journal of Combinatorics, 38 (2014), 12-22.

16. P. Horak and D. Kim, 50 Years of the Golomb-Welch Conjecture, IEEE Transactions on Information Theory, 60 (2018), 3048-3061.

17. M. Livingston and Q. F. Stout, Perfect Dominating Sets, Congr. Numer., 79 (1990), 187-203.

18. F. J. MacWilliams and N. J. A. Sloane, The Theory of Error-Correcting Codes, Elsevier 1977.

19. P. Östergård and W. Weakley, Constructing covering codes with given automorphism, Designs, Codes and Cryptography, 16 (1999), 65-73.

20. P. M. Weichsel, Dominating sets in n-cubes, Journal of Graph Theory, 18 (1994) 479488. 